\title{
Aprendizaje basado en proyectos apoyado en mediaciones virtuales y herramientas informáticas en un curso de producción de aves: estudio de caso en las Ciencias Agropecuarias
}

\section{Project-based learning strategy, supported by virtual mediations and computer tools in a poultry production course: case study in Agricultural Sciences}

\author{
Díaz S Luis ${ }^{1}$ M.Sc, Montes V Donicer ${ }^{1}$ Ph.D
}

${ }^{1}$ Universidad de Sucre, Facultad de Ciencias Agropecuarias, Departamento de Zootecnia, Sincelejo, Colombia.

\section{Keywords:}

Project-based learning; virtual; computer science; Animal Science.
Palabras Clave:

Aprendizaje basado en proyectos;

virtual; informáticas; Zootecnia.

\section{Abstract}

The project-based learning strategy, supported by virtual mediations and computer tools, was applied to 42 students of a program of Animal Science in the course poultry Production in which the degree of familiarization, academic productivity, e-mail asynchronous mediation, the domain of the excel spreadsheet and the appreciation against the implemented methodology. The results showed that $100 \%$ of the students did not know the learning strategy and showed fears at the beginning of the activity. The final grades obtained $(4.44 \pm 0.14,38.09 \% ; 3.67 \pm$ $0.09 ; 38.09 \% ; 2.8,19.05 \%$ ), delivered products and degree of achievement $(100 \%$, 31 students; $88.88 \%, 5$ students; $77.77 \%$, 3 students; $55.55 \%$, 3 students) were influenced by the degree of mastery of the spreadsheet ( 8 students showed mastery, 26 a basic level to elementary and scarce, the rest) and the registered participation level. It was found that the strategy generated motivation in the students reflected in the accomplishment of the goals and objectives drawn at the beginning of the course, increased the student-teacher interaction and reached a high academic performance (final grades $\geq 3.7$ ) in the majority of the participants $(73.8 \%)$.

\section{Resumen}

La estrategia de aprendizaje basado en proyectos, apoyada en mediaciones virtuales y herramientas informáticas fue aplicada a 42 estudiantes de un programa de Zootecnia en la asignatura Producción de Aves en la que se determinó el grado de familiarización, la productividad académica, la mediación asincrónica por medio del correo electrónico, el dominio de la hoja de cálculo excel® y la apreciación frente a la metodología implementada. Los resultados demostraron que el $100 \%$ de los estudiantes no conocían la estrategia de aprendizaje y mostraron temores al inicio de la actividad. Las notas finales obtenidas (4.44 \pm 0.14 , el $38.09 \%$; $3.67 \pm 0.09$, el $38.09 \%$; 2.8 , el $19.05 \%)$, los productos entregados y el grado de cumplimiento (100\%, 31estudiantes; $88.88 \%, 5$ estudiantes; $77.77 \%$, 3 estudiantes; $55.55 \%$, 3 estudiantes) se vieron influenciados por el grado de dominio de la hoja de cálculo (8 estudiantes mostraron dominio, 26 un nivel básico a elemental, y escaso el restante) y el nivel de participación por correo electrónico registrado. Se encontró que la estrategia de aprendizaje genero motivación en los estudiantes, reflejado en el cumplimiento de las metas y objetivos trazados al inicio del curso, incremento la interacción docente- estudiante y alcanzándose un alto rendimiento académico (notas finales $\geq 3.7$ ) en la mayoría de los participantes $(73.8 \%$ ).
Recibido: 02-03-2017

Aceptado: 10-07-2017.

Correspondencia autor:

luis.diaz@unisucre.edu.co 


\section{Introducción}

Los programas de educación superior en Ciencias Agropecuarias tienen en común una marcada formación teórico-práctica que propicia el desarrollo de competencias transversales en los estudiantes, por la aplicación permanente de los conocimientos propios de sus áreas del saber en actividades relacionadas con su quehacer profesional, generándose constantemente ambientes para un aprendizaje significativo. Es allí, donde el aprendizaje basado en proyectos (ABPr) se convierte en una estrategia ideal para el cumplimiento de los objetivos de las asignaturas que conforman sus planes de estudio. Los ABPr se han aplicado con éxito en programas de Medicina, Ingeniería, Pedagogía, entre otros (RODRIGUEZ, E. et al., 2010; MUÑOZ, J. et al., 2011; MALDONADO P, M. 2008). Al ser comparada con metodologías conductistas, este tipo de estrategia muestra un mayor grado de aprendizaje, permite la participación activa del estudiante, mejorando sus resultados, conocimientos y hábitos, desarrolla habilidades como trabajar en equipo, aprendizaje autónomo, responsabilidad y manejo del tiempo (Maldonado, M., 2008).

TRAVERSO, I. et al. (2016), manifiesta que "En las experiencias de aprendizaje basadas en proyectos, la monitorización detallada de las actividades de los miembros de cada equipo puede resultar de utilidad para la evaluación de su trabajo. Mediante procedimientos de evaluación, los supervisores pueden evaluar las capacidades de trabajo en equipo con una finalidad formativa. Suelen aplicarse estrategias como la auto-evaluación, la evaluación entre iguales y la co-evaluación para hacer que la evaluación formativa sea sostenible. Seguir una estrategia de evaluación no es siempre sencillo para los miembros de los equipos, pues necesitan un conocimiento razonable del proceso y los criterios de evaluación", por lo tanto, la rúbrica permite establecer los criterios de evaluación para el proceso de aprendizaje basado en proyectos, ya que a través de los niveles de valoración definidos se emiten un juicios sobre la ejecutoria del proyecto desarrollado por los estudiantes y el nivel alcanzado (MARTINEZ R, J., 2008).
Se espera entonces, que los estudiantes generen productos tangibles derivados de su aprendizaje, ya sea de acuerdo a su área de conocimiento (Zootecnia, Agronomía, Ingeniería), lo que se proyecte (investigación, simulación, proyecciones) o el objeto producido (Artículo científico, maqueta, documento) (BADIA Y GARCIA, 2006).

\section{Metodología}

El estudio de caso se realizó en la granja El Perico ubicada en el Kilómetro 7 vía SincelejoSampués, en las coordenadas $09^{\circ} 15^{\prime}$ de latitud norte y $71^{\circ} 22^{\prime} 54^{\prime \prime}$ de longitud oeste, donde se ubica el campus Ciencias Agropecuarias de la Universidad de Sucre.

La estrategia metodológica de aprendizaje basado en proyectos, apoyada en mediaciones virtuales y herramientas informáticas fue aplicada a 42 estudiantes $(78.57 \%$ hombres y $21.43 \%$ mujeres $)$ de séptimo semestre del programa de Zootecnia en un periodo académico, en la asignatura Producción de Aves I que contempla el desarrollo de competencias profesionales específicas en el manejo, cría, administración, mercadeo y análisis de registros productivos de pollos de engorde para garantizar la productividad, competitividad y sostenibilidad del sistema de producción. La asignatura contempla 2 créditos académicos (Un crédito académico equivale a 48 horas de trabajo del estudiante por semestre.) con una intensidad horaria semanal de $2 \mathrm{~h}$ teóricas y $2 \mathrm{~h}$ prácticas, durante 16 semanas.

Una encuesta para identificar el grado de familiarización y el temor a enfrentarse a la estrategia fue realizada a los estudiantes en la primera sesión, la cual constaba de 2 preguntas; la primera para identificar si conocían y/o habían realizado actividades académicas con este tipo de estrategia metodológica en otras asignaturas de su plan de estudios, con una opción positiva y otra negativa; si la respuesta era afirmativa debía indicar cuales áreas. La segunda fue dirigida para establecer el grado de incertidumbre generado con la propuesta, preguntando si cambiarían la estrategia de aprendizaje teniendo la posibilidad de hacerlo, enunciando con cual, si su respuesta era sí. Surtida esa fase, se socializaron los respectivos objetivos, los productos a generar y la rúbrica evaluativa (Tabla 1). 
Tabla 1. Rúbrica de evaluación acordada con los estudiantes.

\begin{tabular}{|c|c|c|c|c|}
\hline Ítem evaluado & Valoración baja & Valoración media & Valoración alta & $\begin{array}{l}\text { Puntaje } \\
\text { máximo }\end{array}$ \\
\hline $\begin{array}{l}\text { Conformación del } \\
\text { equipo }\end{array}$ & $\begin{array}{l}\text { No hizo parte de ningún } \\
\text { equipo- } \\
0 \text { puntos }\end{array}$ & $\begin{array}{c}\text { Se conformó el equipo, pero no } \\
\text { se asignaron responsabilidades- } \\
\text { Hasta } 10 \text { puntos }\end{array}$ & $\begin{array}{c}\text { Se conformó el equipo } \\
\text { y se distribuyeron } \\
\text { responsabilidades- Hasta } 20 \\
\text { puntos }\end{array}$ & 20 \\
\hline Instalaciones & $\begin{array}{l}\text { No realizaron inspección, ni } \\
\text { participaron en el control de } \\
\text { la limpieza del galpón y/o } \\
\text { no elaboraron el manual de } \\
\text { procedimiento- } \\
0 \text { puntos }\end{array}$ & $\begin{array}{c}\text { Realizo la inspección, o control } \\
\text { de limpieza galpón o elaboro } \\
\text { manual de procedimiento- } \\
\text { Hasta } 40 \text { puntos }\end{array}$ & $\begin{array}{c}\text { Realizo inspección, el } \\
\text { control de desinfección del } \\
\text { galpón y elaboro manual de } \\
\text { procedimiento- } \\
\text { Hasta } 80 \text { puntos. }\end{array}$ & 80 \\
\hline Manejo del pollito & $\begin{array}{l}\text { No realizo ninguna de las } \\
\text { actividades previstas- } \\
0 \text { puntos }\end{array}$ & $\begin{array}{c}\text { Acondiciono o recepción o } \\
\text { controlo y monitorio confort de } \\
\text { los pollitos- } \\
\text { Hasta } 40 \text { puntos }\end{array}$ & $\begin{array}{c}\text { Acondiciono, recepción o, } \\
\text { controlo y monitorio confort de } \\
\text { los pollitos- } \\
\text { Hasta } 80 \text { puntos }\end{array}$ & 80 \\
\hline $\begin{array}{l}\text { Manejo del } \\
\text { crecimiento }\end{array}$ & $\begin{array}{l}\text { No evaluó variabilidad de la } \\
\text { parvada, ni predecir peso vivo } \\
\text { al sacrificio } \\
0 \text { puntos }\end{array}$ & $\begin{array}{c}\text { Evaluó la variabilidad o } \\
\text { monitorio y controlo la } \\
\text { uniformidad o predijo el peso al } \\
\text { sacrificio- } \\
\text { Hasta } 30 \text { puntos }\end{array}$ & $\begin{array}{c}\text { Evaluó variabilidad, monitorio } \\
\text { y controlo la uniformidad y } \\
\text { predijo el peso al sacrificio- } \\
\text { Hasta } 70 \text { puntos }\end{array}$ & 70 \\
\hline Nutrición & $\begin{array}{l}\text { No registra el consumo de } \\
\text { alimento por cada etapa del } \\
\text { ciclo productivo, tampoco } \\
\text { calcula consumo y conversión } \\
\text { alimentaria, ni realiza biometría } \\
\text { del intestino de las aves en } \\
\text { diferentes etapas del ciclo } \\
\text { productivo. } \\
\text { 0 puntos }\end{array}$ & $\begin{array}{l}\text { Registra consumo alimento o } \\
\text { calcula consumo y conversión } \\
\text { alimentaria, o realiza biometría } \\
\text { del intestino de las aves. } \\
\text { Hasta } 30 \text { puntos }\end{array}$ & $\begin{array}{c}\text { Registra consumo alimento, } \\
\text { calcula consumo y conversión } \\
\text { alimentaria y realiza biometría } \\
\text { del intestino de las aves. } \\
\text { Hasta } 70 \text { puntos }\end{array}$ & 70 \\
\hline Higiene y sanidad & $\begin{array}{l}\text { No llevan registro de } \\
\text { vacunación, control sanitario } \\
\text { y mortalidad de las aves, } \\
\text { ni elaboran el manual de } \\
\text { procedimiento. } \\
0 \text { puntos }\end{array}$ & $\begin{array}{l}\text { Llevan registro de vacunación, o } \\
\text { control sanitario o mortalidad de } \\
\text { las aves, o elaboran el manual } \\
\text { de procedimiento. } \\
\text { Hasta } 20 \text { puntos }\end{array}$ & $\begin{array}{l}\text { Llevan registro de vacunación, } \\
\text { control sanitario y mortalidad } \\
\text { de las aves, y elaboran el } \\
\text { manual de procedimiento. } \\
\text { Hasta } 50 \text { puntos }\end{array}$ & 50 \\
\hline $\begin{array}{l}\text { Manejo de aves para } \\
\text { sacrificio }\end{array}$ & $\begin{array}{l}\text { No elaboran un manual de } \\
\text { procedimiento para el manejo } \\
\text { previo de las aves para el } \\
\text { sacrificio, como del destino de } \\
\text { los residuos líquidos y sólidos } \\
\text { de dichas operaciones. } \\
0 \text { puntos }\end{array}$ & $\begin{array}{l}\text { Elaboran un manual de } \\
\text { procedimiento para el manejo } \\
\text { previo de las aves para el } \\
\text { sacrificio, o del destino de los } \\
\text { residuos líquidos y sólidos de } \\
\text { dichas operaciones. } \\
\text { Hasta } 20 \text { puntos }\end{array}$ & $\begin{array}{c}\text { Elaboran un manual de } \\
\text { procedimiento para el manejo } \\
\text { previo de las aves para el } \\
\text { sacrificio, y para el destino de } \\
\text { los residuos líquidos y sólidos } \\
\text { de dichas operaciones. } \\
\text { Hasta } 50 \text { puntos }\end{array}$ & 50 \\
\hline $\begin{array}{l}\text { Índices de } \\
\text { productividad }\end{array}$ & $\begin{array}{c}\text { No medir la productividad en } \\
\text { varias etapas de la producción. } \\
0 \text { puntos }\end{array}$ & $\begin{array}{c}\text { Medir la productividad en una } \\
\text { etapa de la producción. } \\
\text { Hasta } 20 \text { puntos }\end{array}$ & $\begin{array}{l}\text { Medir la productividad } \\
\text { en varias etapas de la } \\
\text { producción. } \\
\text { Hasta } 50 \text { puntos }\end{array}$ & 50 \\
\hline Coevaluación & $\begin{array}{c}\text { No asumió responsabilidades } \\
\text { ni fue coevaluado. } \\
0 \text { puntos }\end{array}$ & $\begin{array}{l}\text { Asumió algunas } \\
\text { responsabilidades o las realizo } \\
\text { deficientemente. } \\
\text { Hasta } 10 \text { puntos }\end{array}$ & $\begin{array}{c}\text { Asumió sus responsabilidades } \\
\text { y coevaluó a su colega. } \\
\text { Hasta } 30 \text { puntos }\end{array}$ & 30 \\
\hline Total & & & & 500 \\
\hline
\end{tabular}


Los productos generados fueron enviados en carpeta comprimida, en formato PDF al correo del docente y devueltos por el mismo medio con las recomendaciones y valoraciones correspondientes. Los registros de consumo, ganancia de peso, control de la uniformidad, predicción del peso al sacrificio, los índices de productividad y sus respectivos gráficos, fueron procesados a través de la hoja de cálculo Microsoft Office Excel ${ }^{\circ}$. Las actividades realizadas por los estudiantes correspondieron al $50 \%$ de la nota final de la asignatura, en donde los 500 puntos máximos posibles eran equivalentes a una valoración de 5.0/5.0.

El impacto de la estrategia metodológica en los estudiantes se relacionó con el porcentaje de cumplimiento de la rúbrica, los productos entregados y la nota obtenida. Otro indicador relevante fueron las actividades de trabajo independiente que realizaron los estudiantes para desarrollar las competencias.

Al final del curso se les realizó una encuesta para establecer el impacto de la propuesta en el proceso de aprendizaje, preguntándoles si les había favorecido o no la estrategia implementada y cuales recomendaciones o ajustes le realizarían.

\section{Resultados}

Las respuestas a la primera pregunta mostraron que el $16.66 \%$ de los estudiantes consideraban la estrategia basada en proyectos como una simulación del sistema de producción, ya que han trabajado en el análisis de datos o registros productivos en otras asignaturas, con el objeto de realizar estimaciones y/o proyecciones del sistema pecuario en estudio, limitándose al aspecto teórico, netamente. El 83.34\% manifestó desconocerla, sin lograr relacionar la estrategia pedagógica propuesta con alguna ya implementada por ellos. Respecto a la segunda pregunta, el $100 \%$ de los estudiantes indico que deseaba cambiar la estrategia por otra conocida y aplicada por los docentes, como seminarios, talleres, informe de prácticas, para mencionar algunas (Tabla 2).
Para el desarrollo de la estrategia, se conformaron 6 grupos de 4 participantes, 3 grupos de 5 participantes y un grupo de 3 participantes, concomitante con el número máximo y mínimo de integrantes preestablecido al inicio del curso. Al analizar los informes entregados a lo largo del semestre, se encontró que el $38.09 \%$ de los estudiantes alcanzó una nota media de $4.44 \pm 0.14$, el $42.85 \%$ una nota media de $3.67 \pm 0.09$ y tan solo el $19.05 \%$ obtuvo una nota reprobatoria de 2.8. El $73.8 \%$ de los estudiantes alcanzaron notas superiores a 3.5 y el $7.14 \%$ una nota igual a 3.0. En la Tabla 3 se relacionan el número de estudiantes por rango de notas obtenidas, los productos entregados y el grado de cumplimiento, teniendo como referencia la rúbrica evaluativa.

Tabla 3. Rango de notas por estudiantes, productos entregados y porcentaje de cumplimiento.

\begin{tabular}{|c|c|c|c|}
\hline $\begin{array}{c}\text { Rango de } \\
\text { notas finales }\end{array}$ & $\begin{array}{l}\text { Número de } \\
\text { estudiantes }\end{array}$ & $\begin{array}{l}\text { Productos } \\
\text { entregados }\end{array}$ & Cumplimiento \\
\hline $0-0.9$ & 0 & N.A & N.A \\
\hline $1,0-1,9$ & 0 & N.A & N.A \\
\hline \multirow{2}{*}{$2,0-2,9$} & 3 & 5 & $55.55 \%$ \\
\hline & 5 & 8 & $88.88 \%$ \\
\hline \multirow{2}{*}{$3,0-3,9$} & 3 & 7 & $77.77 \%$ \\
\hline & 15 & 9 & $100 \%$ \\
\hline $4,0-5,0$ & 16 & 9 & $100 \%$ \\
\hline
\end{tabular}

31 estudiantes cumplieron con el $100 \%$ de los productos (notas $\geq 3.7$ ), asistieron a sesiones extras de acompañamiento dirigido durante el ciclo de producción, reflejando un alto grado de motivación y compromiso por alcanzar los objetivos del proyecto y las metas trazadas en la rúbrica. El grupo de estudiantes (19\%) que no obtuvieron una nota aprobatoria $(\geq 3.0)$, fueron los que mostraron mayor desinterés, menor cumplimiento y bajo o escaso dominio de la hoja de cálculo para analizar datos y generar gráficos. 8 estudiantes demostraron dominio de la hoja de cálculo y 26 un nivel básico a elemental, aspecto que influyo en los productos entregados y la nota obtenida.

Tabla 2. Respuestas a encuesta preliminar.

\begin{tabular}{ccccc}
\hline Respuestas & Pregunta 1 & \multicolumn{2}{c}{ Pregunta 2 } \\
\hline SI & 7 & $16.67 \%$ & 42 & $100 \%$ \\
NO & 35 & $83.33 \%$ & 0 & $0 \%$ \\
& Simulación de la producción en Piscicultura y & Exámenes, talleres y qüices (33 estudiantes) & $78.57 \%$ \\
Cual o & producción bovina (7 estudiantes) & & Seminarios, informe de visitas y clases & $21.43 \%$ \\
cuales & & magistrales (9 estudiantes). & \\
& & & &
\end{tabular}


Las mediaciones virtuales facilitaron la entrega de los productos e incremento la interacción alumno docente, ya que todas las actividades fueron enviadas por ese medio y hubo un feelback que permitió mantener el interés de los estudiantes y enriqueció la experiencia con sus comentarios y aportes. El $100 \%$ de los estudiantes considero que la implementación de la estrategia favoreció su aprendizaje y el $73.8 \%$ recomendó dividir las diversas etapas del ciclo de producción por equipo de trabajo, argumentando que no podían participar todos de cada una de las actividades planeadas.

\section{Discusión}

La encuesta inicial reflejo que el grupo de estudiantes no estaba familiarizado con la estrategia de aprendizaje basada en proyectos y que no había sido implementada en otras asignaturas a largo de su plan de estudios $y$ evidenciando temores relacionados al no cumplimiento de los objetivos propuestos, similar a lo encontrado por MALDONADO, M. (2008) al inicio de la implementación de la estrategia en la asignatura Tecnología de Materiales en un programa de Ingeniería, en donde la comunicación fue deficiente, se presentó dificultad para concretar acuerdos, poca motivación por la desconfianza en los estudiantes para alcanzar los objetivos y mucha incertidumbre.

La libre conformación de los grupos permitió afianzar las relaciones interpersonales y la confianza, permitiéndoles desenvolverse libremente entre pares y aunar esfuerzos para el cumplimiento de los objetivos, excepto en aquellos estudiantes que mostraron el menor porcentaje de cumplimiento, lo que es concordante por lo concluido por GUERRA T, LAURA (2008) en donde los estudiantes de la asignatura Análisis Matemático II establecieron vínculos sociales y de amistad que favorecieron el aprendizaje significativo, debido al apoyo mutuo que les permitió aclarar ideas y afianzar sus conocimientos.

Se evidencio un alto interés de los estudiantes por alcanzar los objetivos y metas planteadas en la rúbrica, ya que un elevado número de estudiantes (31) que participaron de esta estrategia de aprendizaje obtuvieron valoraciones finales altas $(\geq 3.7)$, enviaron los productos en los términos de referencia exigidos, participaban activamente a través del correo electrónico socializando y pidiendo asesoría de sus avances, y emplearon la hoja de cálculo para las actividades requeridas, lo que influyo en el porcentaje de cumplimiento y la nota obtenida. Caso contrario ocurrió con aquellos estudiantes que obtuvieron notas bajas y reprobadas $(\leq 3.0)$, que refuerza lo expuesto por BADIA y GARCIA (2006) que dice: "Los alumnos noveles no suelen tener un conocimiento previo del tema, de los procedimientos y de sus necesidades, y acometen las tareas complejas de forma superficial. Utilizan estrategias poco sofisticadas, no se marcan objetivos ambiciosos y son muy conformistas con el resultado".

El uso de la rúbrica permitió al estudiante establecer el propósito, la metodología, propiciar el trabajo en equipo, cumplir con las metas, interactuar permanentemente con el docente, generar unos productos y socializarlos, cumpliendo con los elementos para el diseño y desarrollo de un proyecto colaborativo descrito por GARCIA y BADIA (2006) y diferenciándose de otros tipo de proyectos acorde a lo expuesto por FIGARELLA (2004), citado por MALDONADO, M. (2008) y opuesto a lo reportado por MUÑOZ y MALDONADO (2011), que aplicaron la estrategia de aprendizaje basado en proyectos para desarrollar las capacidades de problematización en educación superior en un curso de medicina de séptimo semestre de la Universidad Juárez Autónoma de Tabasco, México, en el periodo 2010-1 en la asignatura Proyecto de investigación que les permitiera identificar una problemática de salud de las comunidades de los estudiantes, sus causas y el contexto de prevalencia, centrándose en la búsqueda de información, redacción y entrega de informes, citación bibliográfica y de derechos de autor, encontrando dificultades al inicio del curso por escaso manejo de información en línea, en donde los estudiantes preferían el método expositivo, la memorización y los exámenes, y mostraron poco interés en la estrategia planteada, ya que solo 14 de 31 estudiantes terminaron la actividad.

Al evaluar el impacto en la percepción de la estrategia aplicada sobre el aprendizaje, encontramos que todos los participantes (100\%) la consideraron favorable, ya que el curso permitió la aplicación de los conocimientos adquiridos 
teóricamente y obtener resultados del mismo. Otros casos se han reportado, como el realizado en un programa de ingeniería de Alimentos donde a través de la ejecución de un proyecto para ser presentado en una feria, las encuestas indicaron que el $73 \%$ aprendió a trabajar muy bien en equipo, el $23 \%$ bien y el $3 \%$ considero que fue poco, considerando que el $80 \%$ de los estudiantes expresaron que aprendieron de forma adecuada a planificar, buscar bibliografía, aplicar los conocimientos adquiridos, analizar, interpretar y comunicar resultados y trabajar en grupo (RODRIGUEZ, E. et al., 2010).
Las recomendaciones expuestas por los estudiantes a la estrategia ABP, pudieron deberse a la necesidad de realizar cada etapa del ciclo productivo por grupos separados, reflejando escasas experiencias significativas de aprendizaje colaborativo, pero demostraron y pusieron en práctica habilidades sociales que les permitió trabajar en equipo y manifestar un alto grado de motivación, siendo estos aspectos fundamentales para el cumplimiento de las metas propuestas y el desarrollo de competencias en el área.

\section{Referencias}

BADIA, A. y GARCIA, C. 2006. Incorporación de las TIC en la enseñanza y el aprendizaje basados en la elaboración colaborativa de proyectos. Revista de Universidad y Sociedad del Conocimiento. 3 (2): 42-54. http://www.redalyc.org/articulo.oa?id=78030211 (Consultado el 28 de febrero de 2017)

FIGARELLA, X. 2004. Propuesta para el desarrollo de una metodología orientada a la incorporación de elementos de capacidad emprendedora al diseño de instrucción. Caracas: IV Congreso de Investigación y Creación Intelectual de la UNIMET.

GUERRA T, LAURA. 2008. Estrategias de Aprendizaje Colaborativo utilizando las Nuevas Tecnologías de información y comunicación (Evaluación por grupos). Docencia Universitaria. 9 (2): 21-24

MALDONADO P, M. 2008. Aprendizaje basado en proyectos colaborativos. Una experiencia en educación superior. Revista Educación Laurus. Universidad Pedagógica Experimental Libertador. Venezuela. 14(28): 158-180. http://www.redalyc.org/articulo.oa?id=76111716009 (Consultado el 2 de marzo de 2017).

MARTINEZ R, J. 2008. Las rúbricas en la evaluación escolar: su construcción y uso. Avances en medición. 6: 129-134.

MUÑOZ, J; CÓRDOVA, J; PRIEGO, H. 2011. Aprendizaje con base en proyectos para gestión de riesgos en desastres por estudiantes de medicina. Horizonte Sanitario. 10 (1): 8-20. http://www.redalyc.org/ comocitar.oa?id=457845137004 (Consultado el 8 de noviembre de 2017).

MUÑOZ C, J. y MALDONADO S, T. 2011. Aprendizaje basado en proyectos para desarrollar capacidades de problematización en educación superior. Revista Electrónica Actualidades Investigativas en Educación. Universidad de Costa Rica. 11 (1): 1-19. http://www.redalyc.org/articulo.oa?id=44718060014 (Consultado el 3 de marzo de 2017)

RODRIGUEZ, E; VARGAS, E; LUNA, J. 2010. Evaluación de la estrategia "aprendizaje basado en proyectos". Educación y educadores. 13(1): 13-25

TRAVERSO, I; BALDERAS, A; DODERO, J; RUIZ, I; PALOMO, M. 2016. Evaluación sostenible de experiencias de aprendizaje basado en proyectos. Educación y Cultura en la Sociedad de la Información. 17 (1): $19-43$. 